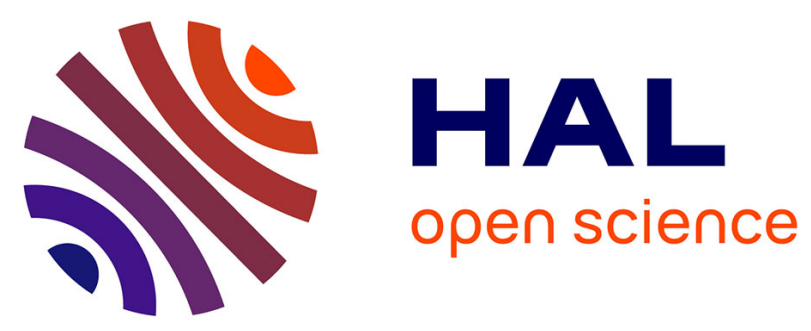

\title{
Effect of pyruvate, lactate and insulin on ATP supply and demand in unpaced perfused rat heart
}

Bernard Korzeniewski, Veronique Deschodt-Arsac, Guillaume Calmettes, Gilles Gouspillou, Jean-Michel Franconi, Philippe Diolez

\section{To cite this version:}

Bernard Korzeniewski, Veronique Deschodt-Arsac, Guillaume Calmettes, Gilles Gouspillou, JeanMichel Franconi, et al.. Effect of pyruvate, lactate and insulin on ATP supply and demand in unpaced perfused rat heart. Biochemical Journal, 2009, 423 (3), pp.421-428. 10.1042/BJ20090395 . hal00479172

\section{HAL Id: hal-00479172 \\ https://hal.science/hal-00479172}

Submitted on 30 Apr 2010

HAL is a multi-disciplinary open access archive for the deposit and dissemination of scientific research documents, whether they are published or not. The documents may come from teaching and research institutions in France or abroad, or from public or private research centers.
L'archive ouverte pluridisciplinaire $\mathbf{H A L}$, est destinée au dépôt et à la diffusion de documents scientifiques de niveau recherche, publiés ou non, émanant des établissements d'enseignement et de recherche français ou étrangers, des laboratoires publics ou privés. 


\section{Effect of pyruvate, lactate and insulin on ATP supply and demand in unpaced perfused rat heart}

Bernard Korzeniewski ${ }^{1,2}$, Véronique Deschodt-Arsac ${ }^{2}$, Guillaume Calmettes ${ }^{2}$, Gilles Gouspillou $^{2}$, Jean-Michel Franconi ${ }^{2}$ and Philippe Diolez ${ }^{2}$

${ }^{1}$ Faculty of Biochemistry, Biophysics and Biotechnology, Jagiellonian University, Kraków, Poland.

${ }^{2}$ Résonance Magnétique des Systèmes Biologiques, UMR5536 Centre National de la Recherche

Scientifique - Université Victor Segalen Bordeaux 2, France.

Correspondence: B. Korzeniewski, Faculty of Biochemistry, Biophysics and Biotechnology, Jagiellonian University, ul. Gronostajowa 7, 30-387 Kraków, Poland, tel. (48 12) 66463 73, fax. (48 12) 66469 02, e-mail: benio@mol.uj.edu.pl

Short title: Effect of pyruvate/lactate and insuline on heart energetics 


\section{SYNOPSIS}

Mitochondrial respiration/oxidative phosphorylation is the main source of energy in the form of ATP in heart under physiological conditions. Different respiratory substrates were used in various experiments during heart perfusion: glucose, pyruvate, lactate, glucose + pyruvate, glucose + lactate, glucose + insulin etc. Also under physiological conditions the concentration of respiratory substrates/hormones in blood can vary significantly. In the present study we tested the effect of pyruvate, lactate and insulin (all in the presence of glucose) and glucose (in the presence of pyruvate) on the ATP-producing and consuming blocks in perfused rat heart, in a system where heart rate (HR) was allowed to vary (no pacing). Changes in rate-pressure product (RPP) and phosphocreatine ( $\mathrm{PCr}$ ) concentration were measured. Proportional Activation Approach (PAA) was used to visualize and quantitatively analyze the data. It was demonstrated that addition of glucose (in the presence of pyruvate) exerted essentially no effect on the system. Insulin (in the presence of glucose) activated only the ATP producer. The most interesting finding is that in our system pyruvate and lactate (added in the presence or instead of glucose) activated ATP producer, but significantly inhibited ATP consumer (their effect was quantitatively identical).

Keywords: oxidative phosphorylation, respiratory substrate, hormone, perfusion, metabolic regulation, Proportional Activation Approach 


\section{INTRODUCTION}

Oxidative phosphorylation in mitochondria is the main source of energy in the form of ATP in heart under physiological conditions. Different respiratory substrates can be used by heart depending on their abundance in blood and on physiological conditions: glucose, pyruvate, lactate, fatty acids, keton bodies. The level of insulin affects significantly the glucose uptake. Different combinations of these substrates/hormones were used as components of the perfusion medium in various experiments on perfused heart: glucose $(G)$, pyruvate $(P)$, lactate $(L)$, glucose + pyruvate (GP), glucose + lactate $(\mathrm{GL})$, glucose + insuline ( $\mathrm{Gl})$ and so on [1-8]. It has been shown [3] that pyruvate elevated RPP and $[\mathrm{PCr}]$ in the paced guinea pig heart system. However, the effects of particular components on the oxidative phosphorylation system have not been systematically studied in unpaced rat heart.

The energetic system of heart can be conceptually divided into two blocks: ATP production and ATP consumption, as it is done within Modular Control Analysis (MoCA) $[5,6]$ or Proportional Activation Approach $[9,10]$. These blocks "communicate" with each other only through a group of metabolites, the concentrations of which are uniquely related to each other: ATP, ADP, $\mathrm{P}_{\mathrm{i}}, \mathrm{PCr}, \mathrm{Cr}$. In principle, each of these metabolites can be chosen as a representative of the entire group, but in practice it is convenient to choose phosphocreatine because its concentration can be measured noninvasively by NMR and it can change significantly (unlike ATP concentration) under physiological conditions. Generally, changes in $\left[\mathrm{PCr}\right.$ ] involve implicitly changes in $[\mathrm{Cr}],[\mathrm{ATP}],[\mathrm{ADP}]$ and $\left[\mathrm{P}_{\mathrm{i}}\right]$. External effectors, such as $\mathrm{Ca}^{2+}$ ions, are not one of the ways in which the PCr-producing block and $\mathrm{PCr}$-consuming block communicate with each other. Heart activation/inactivation results in variations in work and ATP turnover flux that can be represented approximately by rate-pressure product (RPP). Generally, the recording of changes in RPP and [PCr] caused by some external factor enables to find out if ATP producer and consumer are activated or inhibited by this factor, and to which extend $[5,10]$.

It has been demonstrated that both elevated $\left[\mathrm{Ca}^{2+}\right]$ in the perfusion medium $[5]$ and adrenaline [10] activate the ATP producer and consumer to a similar level. However, it was also observed that, in unpaced heart, [PCr] is much higher in heart perfused with pyruvate, than with glucose [5].

Nevertheless, few studies [see e.g. 3,4,11] have been conducted using the same beating hearts on the effect of replacing glucose with pyruvate (or inversely) (or other changes of substrate/hormone composition of the perfusion medium). Respiratory substrate can be considered as an external effector equally well as calcium ions or adrenaline. For instance, it was demonstrated that lactate/pyruvate added to suspension of isolated hepatocytes activate both $\Delta \Psi$ (electrical potential across the inner mitochondrial membrane)-producing and $\Delta \Psi$-consuming system (the former to a slightly greater extent) [9].

It has been demonstrated that Proportional Activation Approach (PAA) [9] is a very useful theoretical method for quantitative analysis of the proportional activation of the producer and consumer of some metabolite (group of metabolites) M by some external effector $[9,10]$. In the present study PAA was used to determine quantitatively the effect (activation or inhibition) on the ATPproducing and ATP-consuming system of the following changes in the substrate/hormone composition of the perfusion medium: 1. replacement of glucose with pyruvate ( $G \rightarrow P$ transition); 2 . addition of pyruvate in the presence of glucose ( $G \rightarrow G P$ transition); 3 . addition of lactate in the presence of glucose ( $G \rightarrow G L$ transition); 4. addition of glucose in the presence of pyruvate (P $\rightarrow G P$ transition); 5 . addition of insulin in the presence of glucose ( $G \rightarrow G$ transition). We hypothesize that the substrate/hormone composition of the perfusion medium has a significant effect on the kinetic properties of the bioenergetic system of the cardiac myocyte.

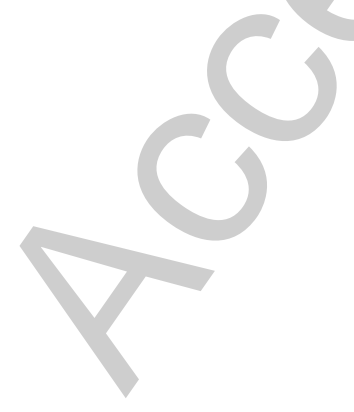




\section{METHODS}

\section{Experimental Procedures}

Langendorf perfusion and NMR measurements procedures for perfused rat (Male SpragueDawley rats : $350-400 \mathrm{~g}$ ) heart were described previously [6]. Hearts were perfused by an aortic canula at constant pressure $(100 \mathrm{~mm} \mathrm{Hg})$ with Krebs-Henseleit solution $\left(95 \% \mathrm{O}_{2}\right.$ and $5 \% \mathrm{CO}_{2}, \mathrm{pH} 7.35$, temperature $37^{\circ} \mathrm{C}$ ) containing $10 \mathrm{mmol} / \mathrm{L}$ Na-pyruvate as substrate and actual free calcium concentration set to $2.0 \mathrm{mmol} / \mathrm{L}$. Left ventricle systolic pressure (LVP) of unpaced heart was measured under isovolumic conditions of contraction thanks to a latex balloon inserted into the left ventricle and connected to a pressure transducer. Mechanical performance was evaluated as the product of heart frequency and developed pressure (RPP, in $\mathrm{mmHg} \cdot$ beat. $\mathrm{min}^{-1}$ ). Generally, we used the unpaced heart system. This differs our system from some other perfusion systems where pacing was applied $[3,4]$. To study the effects of external parameters under physiological conditions, pacing was not applied.

Perfused heart was placed into a $20 \mathrm{~mm}$ NMR tube inserted into a 9.4-T super-conducting magnet equipped with a 20-mm bore (Bruker DPX400 Avance) for 31P NMR spectroscopy. In order to eliminate endogenous substrates and remaining anesthetics, at least 20 minutes were allowed for stabilization of heart activity before any measurement was made.

For the study of steady state modifications four NMR spectra ( 5 min duration time for each spectrum) were acquired before modulation was applied, then after a transition time equivalent to one spectrum (5 $\mathrm{min}$ ) four new spectra $(20 \mathrm{~min})$ were recorded. Each steady state (before and after modulation) was characterized by the mean values of the four spectra for phosphorylated compounds and the corresponding four RPP values.

The effects of the following changes in the substrate/hormone composition of the perfusion medium were studied: $1 . \mathrm{G} \rightarrow \mathrm{P}$ transition, $16.7 \mathrm{mM}$ of glucose was replaced with $10 \mathrm{mM}$ of pyruvate; 2. $\mathrm{G} \rightarrow \mathrm{GP}$ transition, $10 \mathrm{mM}$ of pyruvate was added to $16.7 \mathrm{mM}$ of glucose; 3 . $\mathrm{G} \rightarrow \mathrm{GL}$ transition, 20 $\mathrm{mM}$ of lactate was added to $16.7 \mathrm{mM}$ of glucose; 4 . P $\rightarrow$ GP transition, $16.7 \mathrm{mM}$ of glucose was added to $10 \mathrm{mM}$ of pyruvate; 5 . G $\rightarrow$ GI transition, $20 \mathrm{IU}$ of insulin was added to $16.7 \mathrm{mM}$ of glucose.

The elasticity of the ATP/PCr producer to $\mathrm{PCr}$ can be determined by a direct change of the activity of the consumer. This causes a change in the $\mathrm{PCr}$ concentration which affectes the flux through the producer. The elasticity of the ATP/PCr consumer to PCr can be determined in the opposite way. To perform this kind of analysis one must use agents that directly increase/decrease (modulate) the activity of the consumer and at the same time have no effect on the activity of the producer, or inversely.

$\mathrm{NaCN}$, an inhibitor of cytochrome oxidase, was used to directly decrease the activity of the ATP/PCr producer. The linearity of the response of the consumer to PCr for glucose as the respiratory substrate was tested by applying three different $\mathrm{NaCN}$ concentrations: $0.1,0.2$ and $0.3 \mathrm{mM}$. The obtained results confirmed the assumption concerning the linearity - see Fig. 2, 3 and 4 (compare also the discussion below). The response of the ATP producer to PCr, measured by an increase in the balloon volume in the left ventricle (direct activation of the ATP/PCr consumer), was calculated from averaged relative changes in RPP and [PCr] given in Table 2 in [5] - that experiment was carried out for the same substrate (glucose), rat breed and experimental conditions as the present experiment. The linearity of the response of the ATP/PCr producer to PCr was confirmed in [10] by applying both an increase in the balloon volume in the left ventricle (activation of the consumer) and BDM administration (inhibition of the consumer) (see discussion below). The elasticities of the producer and consumer were calculated as the ratios of the relative changes in RPP to the relative changes in [PCr]: ( $\triangle \mathrm{RPP} / \mathrm{RPP}) /(\triangle \mathrm{PCr} / \mathrm{PCr})$ after an increase in the balloon volume and after $\mathrm{NaCN}$ administration, respectively.

Technically, the measurements of the producer and consumer elasticities to $\mathrm{PCr}$, and of the responses to an external effector must be performed on different hearts.

\section{Theoretical Methods}

The Proportional Activation Approach (PAA) [9] was used in the present work to analyze, interpret and visualize the experimental data. PAA defines the proportional activation coefficient as the ratio of the relative direct activation by some factor $\mathrm{F}$ (it may be e.g. hormone, electrical/neural stimulation or respiratory substrate) of the consumer (C) of some metabolite $\mathrm{M}$ (for instance PCr or ATP) to the relative direct activation of the producer $(P):(\Delta C / C) /(\Delta P / P)[9]$. It was shown [9] that this coefficient equals:

$$
P_{F}^{P C}=\frac{1-\varepsilon_{M}^{C} \cdot \tau}{1-\varepsilon_{M}^{P} \cdot \tau}
$$


where $\varepsilon_{M}^{C}=(\Delta C / C)(\Delta M / M)$ and $\varepsilon_{M}^{P}=(\Delta P / P)(\Delta M / M)$ are elasticities of the consumer and producer to $M$, respectively, while $\tau$ is the response of the system to the factor $F$, defined as the relative change in the metabolite concentration divided by the relative change in flux: $(\Delta M / M) /(\Delta J / J)$.

The values of the elasticities (defined within the so-called Metabolic Control Analysis: see e.g.

[12] for review) of the $\mathrm{M}$ producer and $\mathrm{M}$ consumer blocks to [M] ( $\varepsilon_{M}^{P}$ and $\varepsilon_{M}^{C}$, respectively) quantify the relative change in the flux through these blocks in response to a given relative change in $M$ concentration. The higher the absolute value of the elasticity, the more sensitive is the flux to changes in [M]. In principle elasticities are defined as infinitesimal coefficients, but when the responses of fluxes to $M$ are (nearly) linear, as it takes place in our system, they can be identified with large-scale responses of the producer and consumer blocks to [M]. Under typical conditions $\varepsilon_{M}^{P}$ is negative (M inhibits its own production), while $\varepsilon_{M}^{C}$ is positive (M activates its own consumption).

All possible cases of the effect of some external factor on the system of production and consumption of some metabolite (group of metabolites) $M$ are presented in Fig. 1. When $1 / \tau$ equals $\varepsilon_{M}^{C}$ (the relevant curves overlap) only $\mathrm{P}$ is activated or inhibited, depending on the fact whether flux increases or decreases. When $1 / \tau$ equals $\varepsilon_{M}^{P}$ (the relevant curves overlap) only $\mathrm{C}$ is activated or inhibited. In the special case when $\tau=0$ (no changes in $M$; horizontal line in Fig. 1), both $P$ and $C$ are directly activated or inhibited to the same extent $\left(P_{F}^{P C}=1\right)$. In all other cases both $\mathrm{C}$ and $\mathrm{P}$ are activated and/or inhibited to the relative extent characterized by $P_{F}^{P C}$, as indicated in Fig. 1. In particular, when $P_{F}^{P C}$ is positive both $\mathrm{P}$ and $\mathrm{C}$ are either activated or inhibited, while if $P_{F}^{P C}$ is negative $P$ is activated and $C$ is inhibited, or inversely.

In the present work PAA was used to determine quantitatively the respective direct activation or inhibition of the ATP producer and ATP consumer blocks in the result of changes in the substrate/hormone composition of the perfusion medium, as indicated above.

\section{Statistics and calculations}

Results are presented as means \pm SD. The effects of changes in the substrate/hormone composition of the perfusion medium were compared using one-way ANOVA. Significant differences were considered at $\mathrm{P}<0.05$.

$P_{F}^{P C}$ coefficients were calculated in the following steps. For each particular heart the values of RPP and [PCr] for control (glucose) and modified substrate/hormone composition were used for calculation of the relative (in \%) change in RPP and [PCr] during a given transition. The responses $\tau$ were calculated as indicated above. Proportional activation coefficients were calculated from the responses and elasticities according to Equ. 1 (using the values of the elasticities of the producer and consumer to $[\mathrm{PCr}$ ] equal to -5.34 and 0.96 , respectively, determined as described above). Using this method, only RPP and [PCr] and relative changes (in \%) in these variables can be presented as means \pm SD. 


\section{RESULTS AND DISCUSSION}

\section{Effect of pyruvate, lactate and insulin}

In the present study we measured heart rate (HR), left ventricular pressure (LVP), and thus rate-pressure product (RPP), as well as phosphocreatine ( $\mathrm{PCr}$ ) concentration in the same hearts in control conditions (with glucose as the respiratory substrate) and after a given transition (modification of the substrate/hormone composition in the perfusion medium). The results of these measurements for different transitions are presented in Tables 1 and 2. One can easily see (Table 1) that pyruvate, lactate and insulin caused statistically significant changes in both RPP and [PCr]. Pyruvate and lactate decreased RPP, while insulin increased this variable. In all cases a significant increase in [PCr] was observed. As to the components of RPP, both HR and LVP were affected, although the effect on LVP seems to be generally greater than on HR (Table 2). Averaged relative changes (in \%) in RPP and [PCr] were used for calculation of the responses $\tau$ of the system to modifications of substrate/hormone composition, and subsequently for calculation of proportional activation coefficients (Table 3).

The values of RPP and [PCr] for control conditions (with glucose) were used as reference values and scaled for $100 \%$ for each transition. This is what $100 \%$ (the 'reference point') means in Figs. 2-4. Scaling for $100 \%$ is important because it enables to compare different experiments and Proportional Activation Approach deals with relative changes in fluxes and metabolite concentrations. The values of RPP and [PCr] after particular transitions (modifications in the substrate/hormone composition) were scaled appropriately in relation to this reference point. Relative changes (in \%) in $\mathrm{RPP}$ and [PCr] during particular transitions are shown in first two columns of Table 3.

The data concerning the $G \rightarrow G P$ and $G \rightarrow P$ transitions clearly demonstrated that, in our system, while pyruvate significantly activates the ATP producer, it aiso surprisingly significantly inhibits the ATP consumer. This can be seen in Fig. 2, where both transitions are directed up and left from the initial point (compare Fig. 1). The calculated $P_{F}^{P C}$ coefficients equal -0.307 and -0.296 for $\mathrm{G} \rightarrow \mathrm{GP}$ transition and $G \rightarrow P$ transition, respectively. This means that in both cases the activation of the producer was about three times higher than the inhibition of the consumer. It may seem paradoxical that, although the activation of the producer is about three times greater than the inhibition of the consumer, the flux through the system decreases and not increases. However, one should bear in mind that the consumer has a much greater ( 3.7 times with glucose as the substrate) control over the flux than the producer [5]. The effect on the flux is proportional to the product of the relative activation/inhibition and the control over flux. For this reason in our system the smaller relative inhibition of the consumer prevails over the much higher relative activation of the producer.

We tested both transitions, because, although we used in our previous studies [5] either $G$ or $P$ system, the $G \rightarrow P$ transition comprises the combined effect of pyruvate addition and glucose removal. On the other hand, the $G \rightarrow G P$ transition is equivalent to a pure effect of pyruvate addition. Anyway, both transitions are similar, indicating that the effect of glucose removal (when pyruvate is added) is minor.

This conclusion is supported by the studies on the $P \rightarrow G P$ transition, where no statistically significant effect on either RPP or [PCr] was observed (data not shown). This result strongly suggests that in the presence of saturating concentrations of pyruvate, glucose has negligible influence on the kinetic properties of the system.

The effect of lactate ( $\mathrm{G} \rightarrow \mathrm{GL}$ transition) was very similar to that of pyruvate, although both metabolites exert opposite effect on the cytosolic NADH/NAD ${ }^{+}$ratio. This is demonstrated in Fig. 3. The calculated proportional activation coefficient $P_{F}^{P C}$ for lactate equals - 0.310 . Again, as in the case of pyruvate, this means that the inhibition of the consumer is about three times smaller than the activation of the producer.

In the presence of glucose, insulin activates directly ATP producer and has no direct effect on ATP consumer. This is clearly seen in Fig. 4 where the $G \rightarrow G$ I transition is presented. It is evident that the effect of insulin addition overlaps (within experimental error) with the consumer elasticity to PCr. Thus, the $P_{F}^{P C}$ coefficient equals (approximately) zero (see Table 3). As it is explained in Fig. 1, this fact means a pure activation by insulin of ATP producer without the inhibiting effect on the consumer observed with pyruvate and lactate. In fact, when the metabolic control is shared by both the producer and consumer, a direct activation of the producer only will cause an increase in [PCr], which in turn indirectly activates the consumer and increases RPP. In such a case the slope of the response to some external effector (hormone, substrate) overlaps with the elasticity of the consumer to [PCr], as it can be seen in Fig. 4. In other words, this result simply means that the consumer is not activated directly, but only via the increase in [PCr] caused by the direct activation of the producer. 
Generally, the present results demonstrate that the substrate/hormone composition of the perfusion medium has pronounced impact on the kinetic properties of oxidative phosphorylation in perfused heart.

\section{Mechanism of pyruvate/lactate effect}

The fact that pyruvate/lactate activates the ATP producer is something that could be expected, because as a substrate of the tricarboxic acid cycle it accelerates NADH production in the mitochondrial matrix, elevates the mitochondrial $\mathrm{NADH} / \mathrm{NAD}^{+}$ratio and thus activates oxidative phosphorylation through a faster electron supply to the respiratory chain. However, perhaps the most surprising finding of the present study is the significant inhibition of the ATP consumer by pyruvate and lactate. This inhibition has nothing to do with the cytosolic redox potential, because pyruvate, being the 'oxidized form of lactate', lowers, through the lactate dehydrogenase, the cytosolic NADH/NAD ${ }^{+}$ ratio, while lactate has the opposite effect. It is also not likely that the inhibition of the consumer results from a decrease in calcium transient, because this would also inhibit the producer. Finally, pyruvate/lactate did not change the cytosolic $\mathrm{pH}$ (roughly estimated from the hardly seen $\mathrm{P}_{\mathrm{i}}$ peak in the NMR spectrum). Therefore, it is difficult to determine the mechanism responsible for the inhibition of the PCr producer by pyruvate/lactate. We can only speculate that heart pacing, absent in our system, somehow prevents the inhibition of heart contractile activity by pyruvate/lactate (see below).

Our previous paper [5] and the present study demonstrate that, in our system, the PCr concentration in a heart perfused with pyruvate is much higher than in a heart perfused with glucose. Recently [10] we speculated that with pyruvate the PCr/Cr ratio can be even as high as 10 . (These intuitive expectations are probably somewhat exaggerated - computer simulations using the computer model of oxidative phosphorylation in heart [13] suggest that the $\mathrm{PCr} / \mathrm{Cr}$ ratio for pyruvate of about 2.5 would be able to account for our results [data not shown]). The present study offers a reliable mechanism that could be responsible for the discussed phenomenon. Namely, both activation of ATP producer and inhibition of ATP consumer increase the $\mathrm{PCr} / \mathrm{Cr}$ ratio. The concomitant action of these two effects is able to elevate the $\mathrm{PCr} / \mathrm{Cr}$ ratio to very high levels.

\section{Reasons of lack of glucose effect}

The lack of significant effects on the system of the addition of glucose in the presence of high levels of pyruvate, evidenced in the present study, is not surprising. Glucose is the substrate of glycolysis that produces just pyruvate. Continuous supply of pyruvate constitutes a metabolic 'shortcut' - glycolysis is not needed any more and it can be inhibited (for instance by a high ATP/ADP ratio, linked to a high $\mathrm{PCr} / \mathrm{Cr}$ ratio, or low $\mathrm{P}_{\mathrm{i}}$ and AMP concentration). As it was formulated in [3], in the presence of pyruvate 'the numerous rate-limiting steps in glycolysis are avoided'. It was suggested in [4] that glycolysis is self-limiting at high fluxes by increasing the cytosolic NADH/NAD ${ }^{+}$ratio and thus blocking glyceraldehyde-3-phosphate dehydrogenase. Generally, in the presence of pyruvate glucose loses its significance as a respiratory substrate.

Of course it regains its importance in the absence of pyruvate and other respiratory substrates. In such a case an increase of glucose supply will of course mean a significant activation of the ATP producer. This is most probably the reason why insulin accelerates ATP synthesis, without having any direct influence on ATP usage (Fig. 4). Namely, it is a well known fact that one of the main functions of this hormone is to increase the glucose uptake by cells from blood. Indeed, it was observed that in isolated perfused heart insulin elevates glucose utilization [7]. Even in our system, where glucose concentration in the perfusion medium is high, the effect of insulin is quite significant. Therefore, one could expect a stronger effect at lower, more physiological glucose concentrations.

It is worth to mention that the contribution of different substrates to ATP synthesis and oxygen consumption may change with workload. For instance, in the perfusion medium used in [11] (glucose + insulin, lactate, oleate) lactate and oleate predominated at low workload (with negligible contribution of glucose, in agreement with our results), while after transition to high workload induced by adrenaline lactate and glucose became to have the highest contribution. This suggests that with adrenaline in the perfusate glucose addition in the presence of pyruvate or lactate would have a significant effect in our experimental system.

\section{Effect of pyruvate/lactate - other studies}

It has been shown, using PAA, that in isolated hepatocyte system a mixture of lactate and pyruvate activates both the $\Delta \Psi$-producing and $\Delta \Psi$-consuming block [9]. This finding differs from what we observe in the present study. However, one should bear in mind that the ATP-consuming system is completely different in beating heart and in liver. In particular, one of the most important ATP-using processes in liver is gluconeogenesis that is activated by its substrates, pyruvate and lactate. 
Additionally, gluconeogenesis is very sensitive to the ATP/ADP ratio [14], unlike ATP usage in our system. Finally, hepatocytes are cells isolated from non-excitable tissue. This example demonstrates clearly that it makes little sense to compare the effect of the same factor (lactate/pyruvate) in completely different systems/conditions, and that different tissues may answer in a completely different way to such a factor.

It has been demonstrated by Zweier and Jacobus [3] that pyruvate, when compared with glucose, causes an increase in both [PCr] and RPP. A rough estimation by means of PAA using this effect and our elasticities of the producer and consumer to $\mathrm{PCr}$ leads to the conclusion that in this case pyruvate activated the producer, but did not have any effect on the consumer. Therefore, the authors did not obtain the inhibition of the consumer encountered in our present study. However, the perfusion systems used in both experiments were very different. In particular, in [3] electrically paced guinea pig hearts were used. In our opinion, this difference between [3] and our experiment would be mainly explained by this kind of stimulation. Moreover, we observed [unpublished data] that the kinetic properties could change in regard to the animal species (rat or guinea pigs).

Kobayashi and Neely [4] found that pyruvate and lactate had different effects on the bioenergetic system in perfused heart. However, again, those authors used the paced heart system.

A similar [PCr] (estimated from the ATP/ADP ratio) and RPP (but a significantly higher respiration rate) in hearts perfused with pyruvate in comparison with hearts perfused with glucose were encountered in [15]. Thus, those findings contradict our data. The reason for this discrepancy is unclear. It may be the different $\left[\mathrm{Ca}^{2+}\right]$ in the perfusate: $1.2 \mathrm{mM}$ in [9] and $2.0 \mathrm{mM}$ in our study. In our previous study [5] an increase in $\left[\mathrm{Ca}^{2+}\right]$ in the perfusate from $2.0 \mathrm{mM}$ to $3.5 \mathrm{mM}$ changed to some extend the quantitative properties of the system. This is demonstrated in Fig. 5 where data concerning the $\mathrm{G} \rightarrow \mathrm{P}$ transition for $\left[\mathrm{Ca}^{2+}\right]=3.5 \mathrm{mM}$ extracted from [5] are presented. This figure constitutes only a crude approximation, because the data for glucose and pyruvate were obtained for different hearts. Nevertheless, one can see that in this case RPP slightly increases, which means that the relative inhibition of the consumer is here smaller than for $\left[\mathrm{Ca}^{2+}\right]=2.0 \mathrm{mM}$ (compare Fig. 1). Another possibility can be the fact that the ventricular pressure was imposed by balloon in our experiment and by afterload in [15].

The above comparison of the results of different studies performed on perfused heart in different experimental systems testifies that the properties of the bioenergetic system in heart depend significantly on the experimental conditions. Therefore, the extrapolation of such studies to physiological conditions must be done with caution.

\section{Linearity of response}

For the quantitative analysis performed using the Proportional Activation Approach (PAA) it must be assumed that the response of the $\mathrm{PCr}$ producer and consumer to $\mathrm{PCr}$ is linear. In our previous study [10] we demonstrated that the response of the producer is linear over a very wide range of $\mathrm{PCr}$ concentrations by using an increase in the balloon volume in the left ventricle that activates the consumer, and an administration of BDM that inhibits the consumer. The calculated elasticities of the producer to PCr were similar in both cases. In the present work we demonstrated the linearity of the response of the consumer to $\mathrm{PCr}$ by using three concentrations of $\mathrm{NaCN}$, an inhibitor of the producer (see Figs. 2, 3 and 4). Therefore, the discussed assumption is correct. The inhibitory effect of pyruvate on the $\mathrm{PCr}$ consumer may seem puzzling, but it is a result of our experimental data obtained in our system, and not of the theoretical method (PAA) used for the analysis of the data (as mentioned above, PAA applied to the data obtained in [3] shows no inhibition of the PCr producer by pyruvate in that system).

\section{Mechanism of insulin effect}

In our recent study [10] we demonstrated that adrenaline activates both ATP production and ATP consumption to a similar extent. We hypothesized that this parallel activation was related to an increase in the calcium transient. However, this mechanism cannot work for insulin, because in this case only the ATP/PCr producer is activated (see Fig. 4). An increase in the calcium transient should cause an activation of both the producer and consumer. Therefore, it seems that the mechanisms of the action of adrenaline and insulin are completely different. This is not surprising, because the role of these hormones also seems to be different: adrenaline elevates the energy metabolism and heart work, while insulin only selectively increases the uptake by cardiomyocytes of one of the respiratory substrates: glucose. A similar reasoning holds for the action of pyruvate/lactate: the inhibition of the ATP/PCr consumer testifies that an increase in the calcium transient is not involved in the activation of the producer in this case. 


\section{Implications for control in health and disease}

Our results clearly suggest that heart energetics in vivo is controlled not only by sympathetic/parasympathetic neural system and 'high-energy' hormones such as adrenaline, but also by the substrate composition of blood and the transport of substrates from blood to cardiac myocytes. In particular, pyruvate and lactate are used by heart preferentially in relation to glucose and activate NADH supply to the respiratory chain. The physiological relevance of the inhibition of the ATP consumption by pyruvate/lactate encountered in our unpaced system is not clear, because the heart in vivo is 'paced' by the sympathetic neural system and such inhibition is not seen in electrically paced perfused hearts. An increase in glucose content in blood (taking place e.g. in diabetes) by itself (at high levels of pyruvate/lactate) does not increase the glucose utilization by heart. Glucose uptake from blood is also diminished at low levels of insulin, also taking place in diabetes. This leads to the conclusion that the heart in diabetic individuals cannot use the excess of glucose as a preferential respiratory fuel and therefore contribute to a decrease in its concentration in blood. Perhaps this restriction could be overcome by lowering the pyruvate/lactate concentration in blood and/or increase in the adrenaline level.

\section{Summary}

Summing up, in the present study we demonstrate that the substrate/hormone composition of the perfusion medium has a pronounced influence on the kinetic properties of oxidative phosphorylation in perfused mammalian heart. In our system, with no heart pacing, pyruvate and lactate activate the ATP-producing block, but at the same time they significantly inhibit the ATPconsuming block. Glucose exerts essentially no effect in the presence of high pyruvate concentrations. Insulin (in the presence of glucose) activates only the ATP producer, probably through stimulating glucose uptake from the perfusion medium. Generally, our data suggest that various hormones (adrenaline and insulin) have very different mechanisms of action. It appears interesting to study the combined effects of physiological modulators of cardiac energetics in healthy and pathological hearts. 


\section{REFERENCES}

1. From, A. H., Petein, M.A., Michurski, S.P., Zimmer, S.D. and Uğurbil, K. (1986) ${ }^{31}$ P-NMR studies of respiratory regulation in the intact myocardium. FEBS Lett. 206, 257-61

2. From, A.H.L., Zimmer, S.D., Michurski, S.P., Mohanakrishnan, P., Ulstad, V.K., Thoma, W.J. and Ugurbil, K. (1990) Regulation of the oxidative phosphorylation rate in the intact cell. Biochemistry 29, 3731-3743

3. Zweier, J.L. and Jacobus, W.E. (1987) Substrate-induced alterations of high energy phosphate metabolism and contractile function in the perfused heart. J. Biol. Chem. 262, 8015-8021.

4. Kobayashi, K. and Neely, J.R. (1979) Control of maximum rates of glycolysis in rat cardiac muscle. Circ. Res. 44, 166-175.

5. Diolez, P., Descholdt-Arsac, V., Raffard, G., Simon, C., Dos Santos, P., Thiaudiere, E., Arsac, L. and Franconi, J.-M. (2007) Modular regulation analysis of heart contraction: application to in situ demonstration of a direct mitochondrial activation by calcium in beating heart. Am. J. Physiol. 293, R13-R19

6. Diolez, P., Raffard, G., Simon, C., Leducq, N., Dos Santos, P. and Canioni, P. (2002) Mitochondria do not control heart bioenergetics. Molecular Biology Reports 29, 193-196

7. Kashiwaya, Y., Sato, K., Tsuchiya, N., Thomas, S., Fell, D.A., Veech, R.L. and Passonneau, J.V. (1994) Control of glucose utilization in working perfused rat heart. J. Biol. Chem. 269, 25502-25514 8. Sharma, N., Okere, I.C., Brunengraber, D.Z., McElfresh, T.A., King, K.L., Sterk, J.P., Huang, H., Chandler, M.P. and Stanley, W.C. (2005) Regulation of pyruvate dehydrogenase activity and citric acid cycle intermediates during high cardiac power generation. J. Physiol. 562, 593-603

9. Korzeniewski, B., Harper, M.-E. and Brand, M.D. (1995) Proportional activation coefficients during stimulation of oxidative phosphorylation by lactate and pyruvate or by vasopressin. Biochim. Biophys. Acta 1229, 315-322

10. Korzeniewski, B., Deschodt-Arsac, V., Calmettes, G., Franconi, J.-M. and Diolez, P. (2008) Physiological heart activation by adrenaline involves parallel activation of ATP usage and supply. Biochem. J. 413, 343-347

11. Dos Santos. P., Aliev, M.K., Diolez, P., Duclos, F., Besse, P., Bonoron-Adele, S., Sikk, P., Canioni, P. and Saks, V.A. (2000) Metabolic control of contractile performance in isolated perfused rat heart. Analysis of experimental data by reaction:diffusiom mathematical model. J. Mol. Cell. Cardiol. 32, 1703-1734

12. Fell, D.A. (1992) Metabolic Control Analysis: a survey of its theoretical and experimental development. Biochem. J. 286, 313-330.

13. Korzeniewski, B., Noma, A. and Matsuoka, S. ( 2005) Regulation of oxidative phosphorylation in intact mammalian heart in vivo. Biophys. Chem. 116, 145-157

14. Buttgereit, F. and Brand, M.D. (1995) A hierarchy of ATP-consuming processes in mammalian cells. Biochem. J. 312,163-167

15. Jeffrey, F. M. and Malloy, C.R. (1992) Respiratory control and substrate effects in the working rat heart. Biochem. J. 287, 117-123 
B Biochemical Journal Immediate Publication. Published on 18 Aug 2009 as manuscript BJ20090395

\section{ACKNOWLEDGEMENTS}

This work was supported by the AFM (Association Française contre les Myopathies) and the Region Aquitaine, France 
Table 1. Mechanical performance of hearts (rate-pressure product, RPP) and phosphocreatine concentrations ([PCr]) for control hearts (with glucose) and modified substrates/hormone compositions.

\begin{tabular}{lllll}
\hline $\begin{array}{l}\text { transition } \\
\text { (number of hearts) }\end{array}$ & $\begin{array}{l}\text { RPP }\left(\mathrm{mmHg} \cdot{\text { beat. } \mathrm{min}^{-1}} \text { ) }\right. \\
\text { (mean } \pm \mathrm{SD}) \\
\text { control } \\
\text { (glucose) }\end{array}$ & $\begin{array}{l}\text { after } \\
\text { transition }\end{array}$ & $\begin{array}{l}\text { [PCr] }(\mathrm{mM}) \\
(\text { mean } \pm \mathrm{SD}) \\
\text { control } \\
\text { (glucose) }\end{array}$ & $\begin{array}{l}\text { after } \\
\text { transition }\end{array}$ \\
\hline $\mathrm{G} \rightarrow \mathrm{P}(\mathrm{n}=7)$ & 48031 & 35683 & 10.71 & 17.16 \\
& \pm 9483 & $\pm 3783^{* *}$ & \pm 1.00 & $\pm 1.27^{* * *}$ \\
$\mathrm{G} \rightarrow \mathrm{GP}(\mathrm{n}=6)$ & 43879 & 33753 & 11.35 & 16.28 \\
& \pm 6687 & $\pm 1956^{* * *}$ & \pm 1.30 & $\pm 0.71^{* * *}$ \\
$\mathrm{G} \rightarrow \mathrm{L}(\mathrm{n}=5)$ & 48075 & 40195 & 13.3 & 17.6 \\
$\mathrm{G} \rightarrow \mathrm{Gl}(\mathrm{n}=6)$ & \pm 5974 & $\pm 3634^{* *}$ & \pm 0.6 & $\pm 1.0^{* * *}$ \\
& 50734 & 57347 & 14.03 & 16.12 \\
& \pm 2298 & $\pm 1097^{* *}$ & \pm 1.17 & $\pm 1.35^{* * *}$ \\
\hline
\end{tabular}

${ }^{* *} \mathrm{P}<0.01$ between control (glucose) and modified substrates/hormone composition ${ }^{* * *} P<0.001$ between control (glucose) and modified substrates/hormone composition 
Table 2: Heart Rate (HR) and left ventricle pressure (LVP) for control hearts (with glucose) and modified substrates/hormone compositions.

\begin{tabular}{|c|c|c|c|c|}
\hline $\begin{array}{l}\text { transition } \\
\text { (number of hearts) }\end{array}$ & $\begin{array}{l}\mathrm{HR}\left(\text { beat } \min ^{-1}\right) \\
(\text { mean } \pm \mathrm{SD}) \\
\text { control } \\
\text { (glucose) }\end{array}$ & $\begin{array}{l}\text { after } \\
\text { transition }\end{array}$ & $\begin{array}{l}\text { LVP }(\mathrm{mmHg}) \\
\text { (mean } \pm \mathrm{SD}) \\
\text { control } \\
\text { (glucose) }\end{array}$ & $\begin{array}{l}\text { after } \\
\text { transition }\end{array}$ \\
\hline $\mathrm{G} \rightarrow P(\mathrm{n}=7)$ & $\begin{array}{l}285 \\
\pm 23\end{array}$ & $\begin{array}{l}258 \\
\pm 25 \text { * }\end{array}$ & $\begin{array}{l}172 \\
\pm 16\end{array}$ & $\begin{array}{l}138 \\
\pm 19\end{array}$ \\
\hline $\mathrm{G} \rightarrow \mathrm{GP}(\mathrm{n}=6)$ & $\begin{array}{l}241 \\
\pm 39\end{array}$ & $\begin{array}{l}222 \\
\pm 20\end{array}$ & $\begin{array}{l}194 \\
\pm 2\end{array}$ & $\begin{array}{l}150 \\
\pm 32\end{array}$ \\
\hline$G \rightarrow L(n=5)$ & $\begin{array}{l}300 \\
\pm 16\end{array}$ & $\begin{array}{l}292 \\
\pm 35\end{array}$ & $\begin{array}{l}166 \\
\pm 15\end{array}$ & $\begin{array}{l}136 \\
\pm 17 \text { *** }\end{array}$ \\
\hline $\mathrm{G} \rightarrow \mathrm{GI}(\mathrm{n}=6)$ & $\begin{array}{l}256 \\
\pm 16\end{array}$ & $\begin{array}{l}241 \\
\pm 7\end{array}$ & $\begin{array}{l}178 \\
\pm 25\end{array}$ & $\begin{array}{l}238 \\
\pm 8^{* *}\end{array}$ \\
\hline
\end{tabular}

${ }^{*} \mathrm{P}<0.05$ between control (glucose) and modified substrates/hormone composition
${ }^{* *} \mathrm{P}<0.01$ between control (glucose) and modified substrates/hormone composition
${ }^{* * *} \mathrm{P}<0.001$ between control (glucose) and modified substrates/hormone composition 
Table 3. Averaged relative changes (in \%) in RPP and [PCr] as well as calculated responses and proportional activation coefficients during transition from control (glucose) to modified substrate/hormone composition.

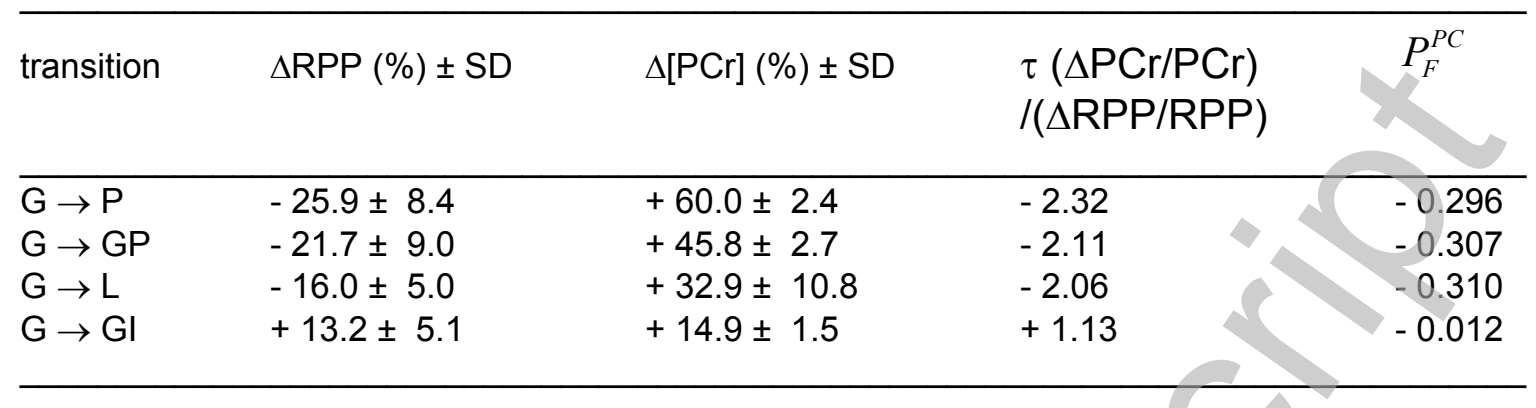




\section{FIGURE LEGENDS}

Fig. 1. Potential possibilities of activation/inhibition by an external effector of producer and consumer of an intermediate metabolite $\mathrm{M}$ within Proportional Activation Approach. $100 \%$ for flux and [M] corresponds to the initial state (before the action of the external effector), while the area surrounding this point represents all possibilities of external action. When the effect overlaps with the elasticity of the consumer to $\mathrm{M}\left(\varepsilon_{\mathrm{C}}\right)$, only the producer is activated/inhibited. When the effect overlaps with the elasticity of the producer to $M\left(\varepsilon_{\mathrm{P}}\right)$, only the consumer is activated/inhibited. When the effect lies somewhere between $\varepsilon_{C}$ and $\varepsilon_{P}$, both producer and consumer are affected (activated or inhibited), as indicated in the figure.

Fig. 2. Effect of $\mathrm{G} \rightarrow \mathrm{P}$ and $\mathrm{G} \rightarrow \mathrm{GP}$ transition. $\varepsilon_{\mathrm{C}}$, elasticity of consumer to [PCr] determined by $\mathrm{NaCN}$ addition; $\varepsilon_{P}$, elasticity of producer to [PCr] determined by increase in balloon volume; Gluc, glucose; Pyr, pyruvate. It can be clearly seen that pyruvate activates ATP producer, but inhibits ATP consumer.

Fig. 3. Effect of $\mathrm{G} \rightarrow \mathrm{GL}$ transition. $\varepsilon_{\mathrm{C}}$, elasticity of consumer to [PCr] determined by NaCN addition; $\varepsilon_{\mathrm{P}}$, elasticity of producer to [PCr] determined by increase in balloon volume; Gluc, glucose. It can be clearly seen that lactate activates ATP producer, but inhibits ATP consumer.

Fig. 4. Effect of $\mathrm{G} \rightarrow \mathrm{GI}$ transition. $\varepsilon_{\mathrm{C}}$, elasticity of consumer to [PCr] determined by NaCN addition; $\varepsilon_{\mathrm{P}}$, elasticity of producer to [PCr] determined by increase in balloon volume. It can be clearly seen that insulin activates only ATP producer.

Fig. 5. Effect of $\mathrm{G} \rightarrow \mathrm{GP}$ transition for high ( $3.5 \mathrm{mM}$ ) calcium ion concentration in perfusate: data extracted from [2]. Gluc, glucose; Pyr, pyruvate. It can be clearly seen that pyruvate activates ATP producer and inhibits ATP consumer; however, the inhibition of consumer is smaller than for low (2.0 $\mathrm{mM}$ ) calcium ion concentration in perfusate measured in the present study (compare Fig. 2). 
Fig. 1

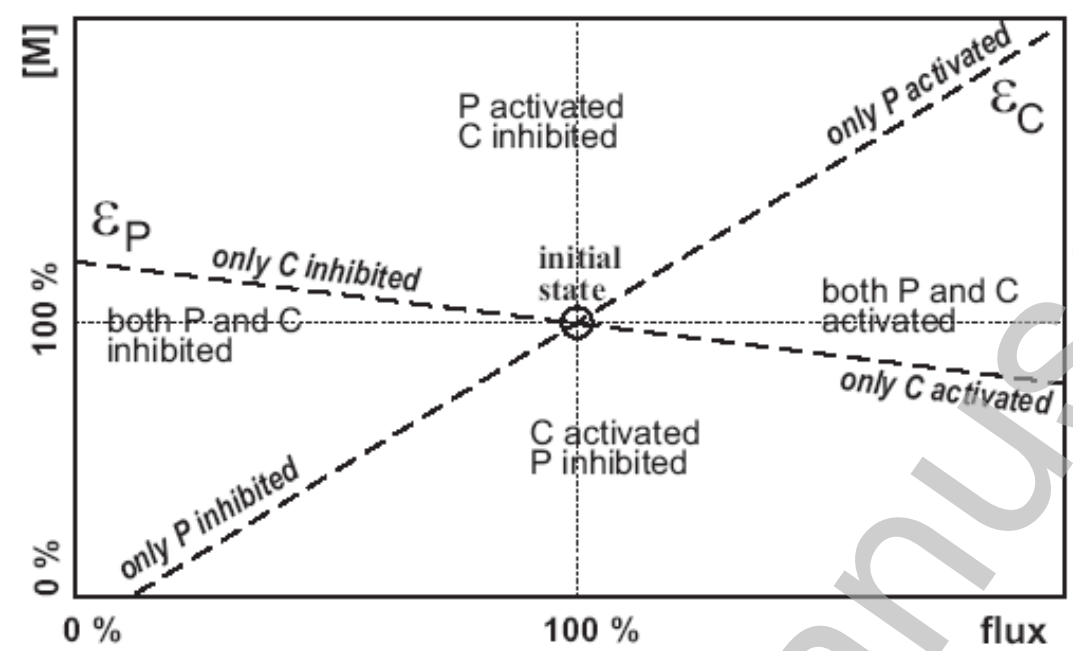


Fig. 2

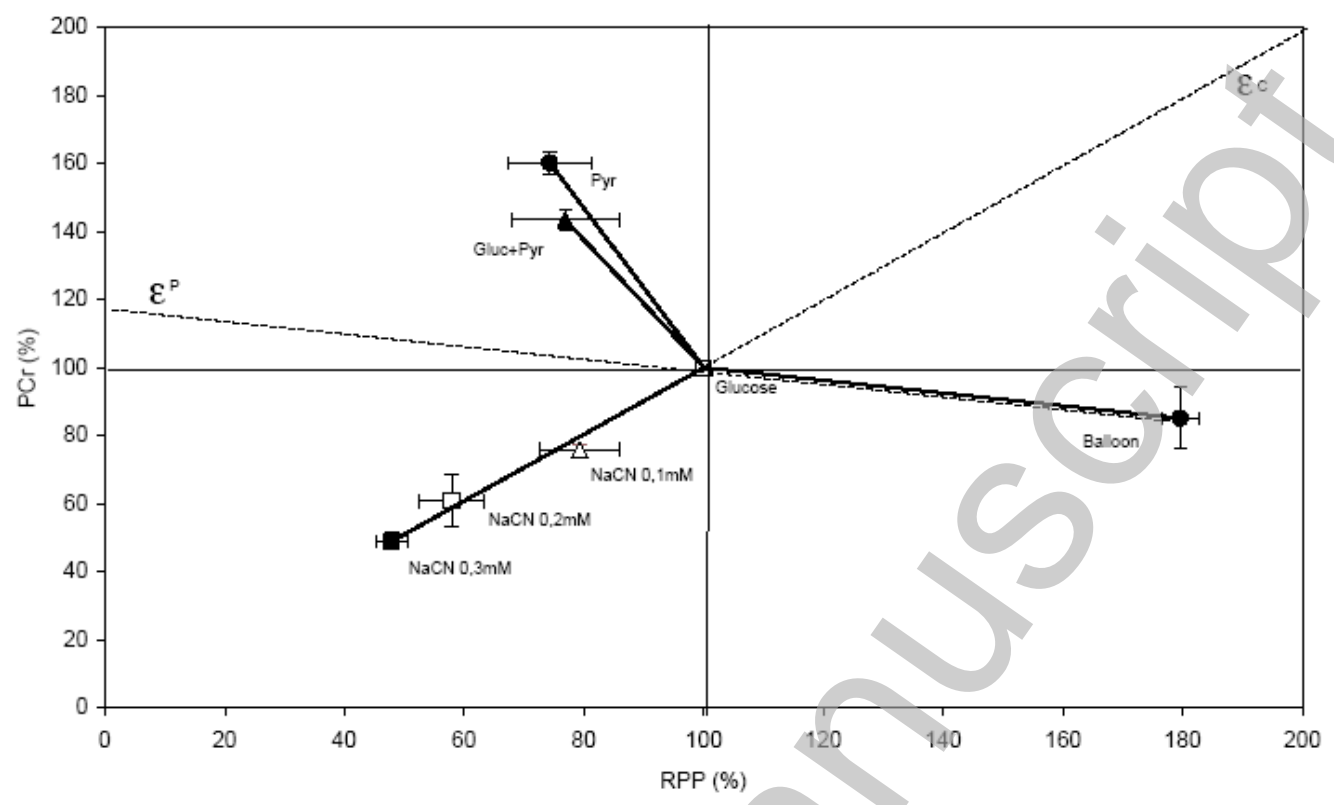

Licenced copy. Copying is not permitted, except with prior permission and as allowed by law. (C) 2009 The Authors Journal compilation (c) 2009 Portland Press Limited 
Fig. 3

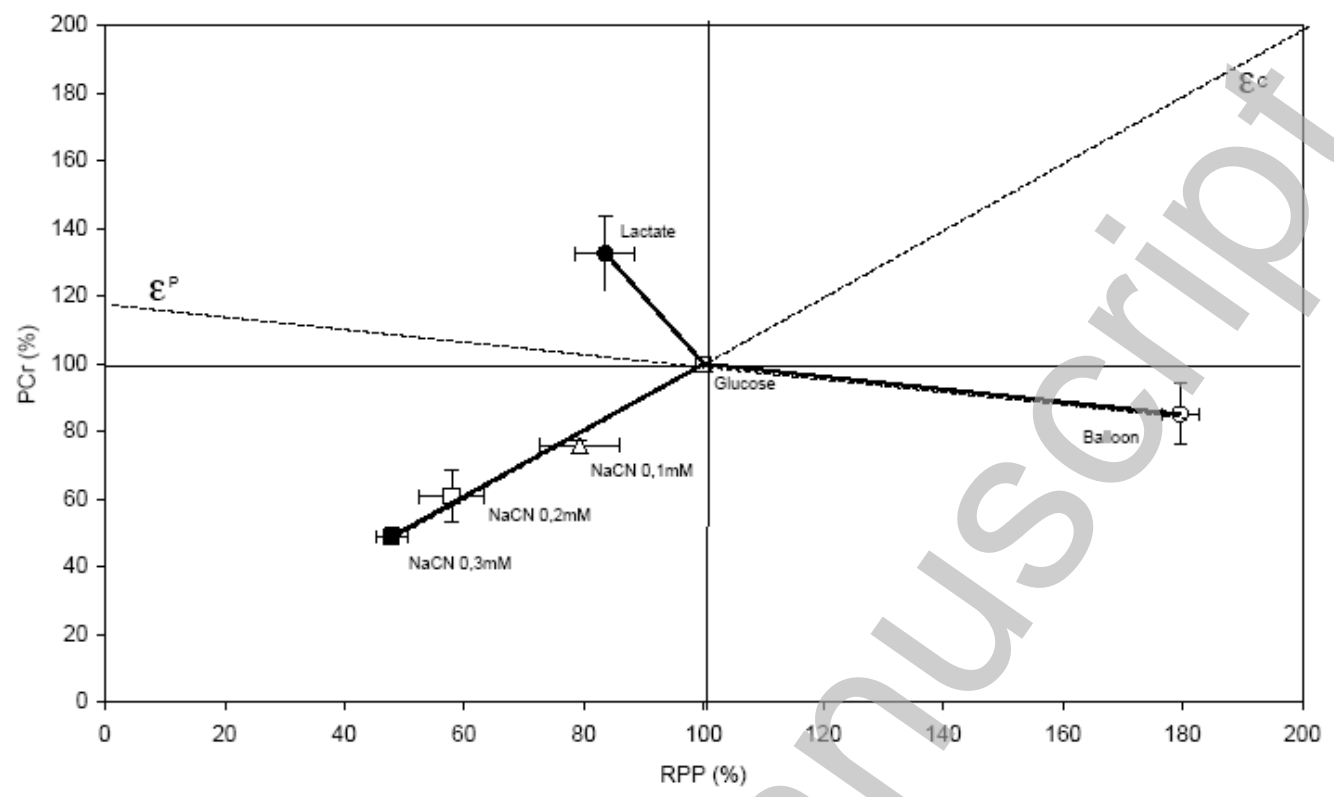

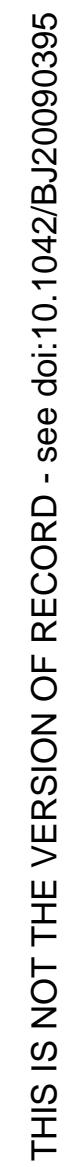


Fig. 4

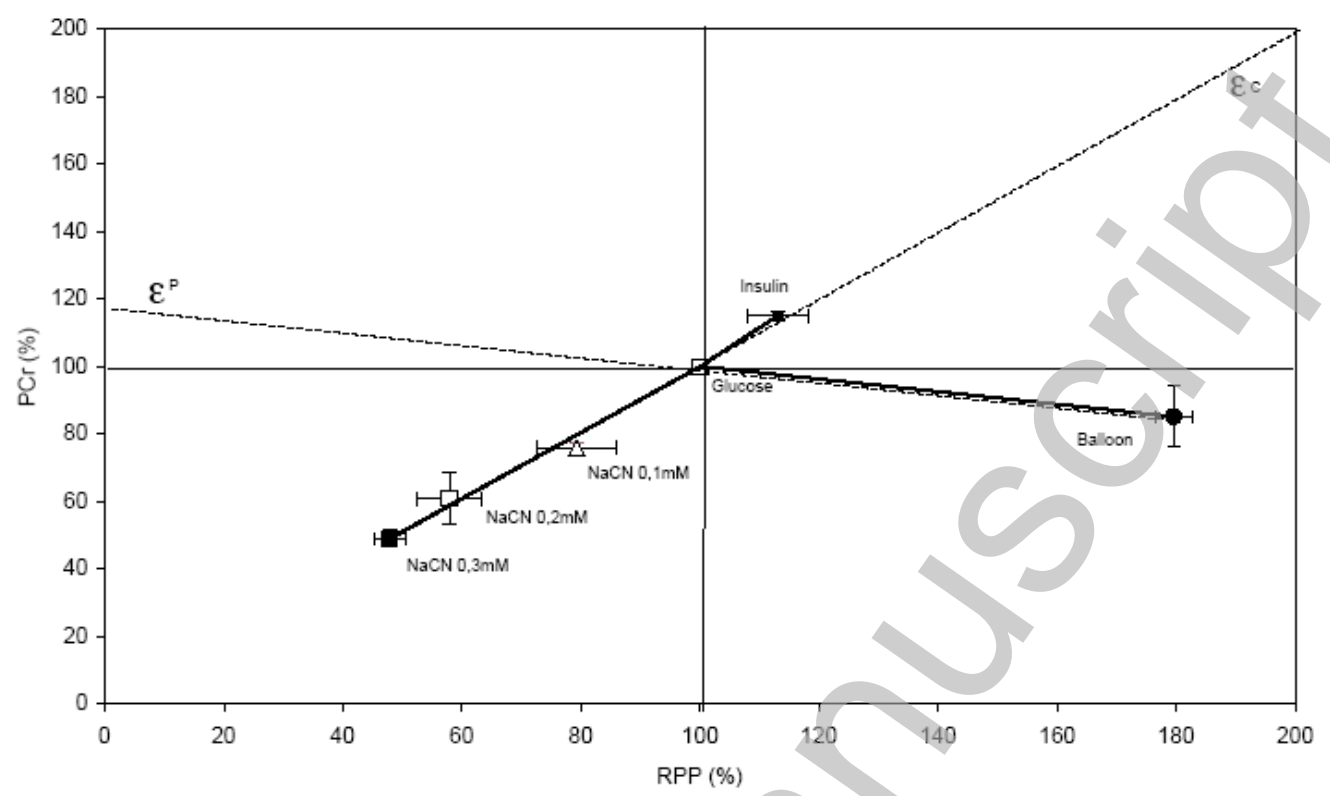

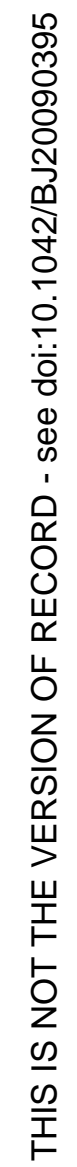


Fig. 5

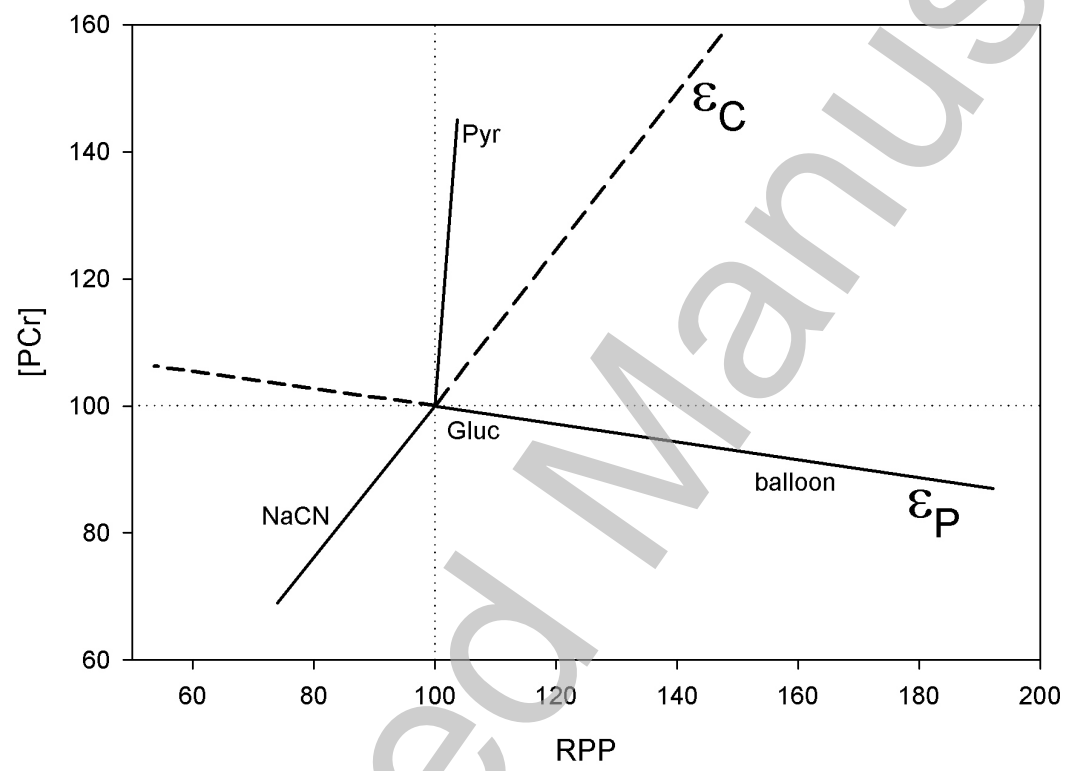

